\title{
Authors' reply: Systematic literature analysis and review of targeted preventive measures to limit healthcare-associated infections by meticillin-resistant Staphylococcus aureus
}

\author{
R Köck ${ }^{1}$, A W Friedrich (alex.friedrich@umcg.nl)2 ${ }^{2}$ on behalf of the original author group ${ }^{3}$ \\ 1. Institute of Hygiene, University Hospital Münster, Münster, Germany \\ 2. Department of Medical Microbiology, University of Groningen, University Medical Center Groningen, Groningen, The \\ Netherlands \\ 3. The members of the group are listed at the end of the article
}

Citation style for this article:

Köck R, Friedrich AW, on behalf of the original author group. Authors' reply: Systematic literature analysis and review of targeted preventive measures to limit healthcare-associated infections by meticillin-resistant Staphylococcus aureus. Euro Surveill. 2014;19(37):pii=20902. Available online: http://www. eurosurveillance.org/ViewArticle.aspx?Articleld=20902

Article submitted on 10 September 2014 / published on 18 September 2014

\section{To the editor:}

We thank Prof. Verbrugh for the letter in regard to our article Systematic literature analysis and review of targeted preventive measures to limit healthcare-associated infections by meticillin-resistant Staphylococcus aureus (MRSA) [1]. As mentioned by Prof. Verbrugh, the article describing the United States Veteran Affairs (VA) initiative to combat MRSA [2] was discussed in our review, but not included in the body of the text and not reviewed in detail. It should clearly be stated that we highly acknowledge the work of the VA initiative. This is why the results of this 'bundle approach' for MRSA prevention were highlighted in the discussion. However, in the literature review, we focused on assessing specifically the impact of three defined single measures (i.e. screening, isolation in single rooms and decolonisation) on the occurrence of MRSA. The question of interest with respect to MRSA screening was: 'Does screening of patients before or on admission reduce the incidence of MRSA infection or transmission? How do PCR-based rapid tests for the direct detection of MRSA from screening specimens influence the incidence of MRSA colonisation or infection compared with culture-based methods?'

In the VA initiative, screening followed by contact precautions was among the measures implemented which, indeed, made the study relevant for consideration in the review. However, the article was excluded from formal review. The main reason was that different microbiological techniques (culture-based and PCR rapid test) were used for MRSA screening in the participating 153 hospitals and results were not stratified between these different methods. This prevented basic categorisation of the article according to the question of interest mentioned above (PCR vs culture). We agree that this formal aspect could have been better explained in the section defining exclusion criteria.
However, we also agree that both the fact that the VA initiative was performed as a real-life intervention (rather than a (randomised) controlled study) and that screening was implemented in a bundle together with 'institutional culture change' and hand hygiene programmes, did not make the initiative irrelevant for learning about MRSA prevention. On the contrary, as we were aware that most evidence evaluating the effects of screening is from observational studies, we explicitly did not restrict data analysis to randomised controlled trials. In addition, we think that the evaluation of care bundles is an important (and feasible) method to assess preventive effects in defined healthcare systems or clinical settings.

Members of the group

K Becker, B Cookson, JE van Gemert-Pijnen, S Harbarth, J Kluytmans, M Mielke, G Peters, RL Skov, MJ Struelens, E Tacconelli, W Witte.

Conflict of interest

None declared.

Authors' contributions

RK, AWF, KB, BC, JEvGP, SH, JK, MM, GP, RLS, MJS, ET and WW replied to the letter.

References

1. Köck R, Becker K, Cookson B, van Gemert-Pijnen JE, Harbarth $\mathrm{S}$, Kluytmans J, et al. Systematic literature analysis and review of targeted preventive measures to limit healthcare-associated infections by meticillin-resistant Staphylococcus aureus. Euro Surveill. 2014;19(29): pii=20860.

2. Jain R, Kralovic SM, Evans ME, Ambrose M, Simbartl LA, Obrosky DS, et al. Veterans Affairs initiative to prevent methicillin-resistant Staphylococcus aureus infections. $\mathrm{N}$ Engl J Med. 2011;364(15):1419-30. http://dx.doi.org/10.1056/ NEJMoa1007474 